\title{
Classifying Human Emotional States using Wireless EEG based ERP and Functional Connectivity Measures
}

\author{
Valentina Bono, Dwaipayan Biswas, Saptarshi Das and Koushik Maharatna, Member, IEEE
}

\begin{abstract}
In this paper we present a systematic exploration to determine several EEG based features for classifying three emotional states (happy, fearful and neutral) pertaining to face perception. EEG data were acquired through a 19-channel wireless system from eight adults under two conditions - in a constrained position and involving head-body movements. The movement EEG data was pre-processed using an artifact reduction algorithm and both datasets were processed to extract neurophysiological features - ERP components and from functional connectivity measures. The functional connectivity measures were processed using a brain connectivity toolbox and gray level co-occurrence matrices to generate a total of $\mathbf{4 6 3}$ features. The feature set was split into: training dataset comprising of constrained and movement EEG data and test dataset comprising of only movement EEG data. A retrospective cross-validation approach was run on the training dataset in conjunction with two classifiers (LDA and SVM) and the ranked feature set, to select the best features using a sequential forward selection algorithm. The best features were further used to prospectively classify the three emotions in the test dataset. Our results show that we can successfully classify the emotions using LDA with an accuracy of $89 \%$ and using top 17 ranked features.
\end{abstract}

\section{INTRODUCTION}

An understanding of the underlying mechanism involved in the perception of different facial expressions incurring a high mental activity has considerable applications in the field of psychology (e.g. studying mood and emotions). Emotional states have been known to be associated with specific psychological response patterns which have been investigated by the research community for various applications in healthcare [1], affecting computing [2] and learning [3]. Out of the several common measures used for emotion recognition [4], neurophysiological measurement can access the processes in the fundamental brain structures responsible for the evolution in emotion dynamics and can hence be used for recognition of a wide range of emotional states.

In particular, EEG with its high temporal resolution, can detect the immediate responses to emotional stimuli [5] and hence various EEG features are implicated in emotion processes. These features at a single electrode level are: 1) components of event related potentials (ERPs) [6], 2) spectral power in different frequency bands and 3) from multichannel perspective - phase synchronization and coherence [7]. As the emotional process involves a large-scale network instead of a single brain region [4], a multichannel EEG analysis

*Research supported by FP7 EU funded MICHELANGELO project, Grant Agreement \#288241.

V. Bono, D. Biswas, S. Das and K. Maharatna are with the Electronics and Computer Science, University of Southampton, Hampshire, SO17 1BJ, UK (e-mail: \{vb2a12, db9g10, sd2a11,km3\}@ecs.soton.ac.uk). investigating the interaction among different brain sites could formulate an understanding of the underlying emotional processes. The information exchange between the network of segregated functional units of the brain which integrate with each other can be described by functional connectivity (FC) measures during emotion processes These measures can be quantified by a number of neuro-biological features using complex network analysis [8].

In this study we aim to investigate several EEG-based features extracted from brain signals acquired during face emotional stimuli to determine the significant features (ERP components and $F C$ measures specific to emotion processing) involved in cognitive processing for face perception. These identified features can be used to classify the emotional states thereby aiding the diagnosis and treatment of patients affected by neurodegenerative diseases, having impaired face emotion recognition (e.g. Autism Spectrum Disorder [1]).

For this investigation, we recorded EEG data elicited by neutral and emotional faces (happiness and fearful) with the subject: in a constrained position (condition1-constrained $E E G$ ) and in real-life involving body/head movements (condition2-movement EEG). A wireless 19-Channel EEG system was used to collect data from the subjects and relevant processing was done to identify robust EEG features required for classifying the different emotional states. The processing hierarchy involved filtering the captured signals and an additional artifact reduction algorithm was applied only on the movement EEG data using wavelet packet transform-empirical mode decomposition (WPT-EMD) [9]. The processed EEG data were averaged across multiple stimuli (26 stimuli were presented for each of the three emotions) to generate the ERP data for the feature extraction of: 1) ERP components and 2) $F C$ based measures. Each $F C$ measure was represented with a reduced dimensionality by applying: 1) gray level co-occurrence matrices (GLCM) [10] and 2) Brain Connectivity Toolbox (BCT). Out of the 463 features extracted, the significant features were selected from a ranked list, using sequential forward selection (SFS) algorithm in conjunction with two classifiers following a cross-validation technique. Our results show that we are able to prospectively classify the three emotional states from condition 2 with an accuracy of $89 \%$ using only 17 features with the Linear Discriminant Analysis (LDA) classifier.

\section{BACKGROUND}

Recent studies have focused on recognizing emotions from neural response using EEG signal features and classifying emotions elicited by pictures, audio and video. Majority of these EEG features are based on wavelet [11] and Hilbert [12] transform. In [5], few brain connectivity 
measures (correlation, coherence, and phase synchronization) have been used to classify positive, neutral and negative emotions elicited by video-clip, which to the best of our knowledge appears to be the most relevant work for emotion recognition.

In our study, we aimed at using neurophysiological features that are deemed to aid in the investigation of the underlying brain processes involved in emotion perception. ERP components and functional connectivity have been commonly used for studies involving face perception in typical and pathological subjects. Here, in addition to the ERP components, we make an attempt to describe these $F C$ measures with a reduced dimensionality by using $B C T$ and GLCM. Finally, we use these features to classify the emotions using a low-complexity classifier.

\section{EEG DATA ACQUISITION}

In this study we chose three common emotional states [5], having a wide range of variability that can trigger diverse brain responses to the applied stimuli. An EEG face-evoked dataset [13] was used consisting of greyscale images of 13 adults (seven males and six females) with three different expressions (happy, fearful and neutral). The external facial features, like hair, neck and ears, were deleted from the stimuli to ensure subject's attention on the facial expression. EEG data were collected under two conditions from eight subjects, who gave consent for the experiments, comprising of two females and six males (mean age of $28 \pm 3$ ). The participants were seated approximately $80 \mathrm{~cm}$ from a computer monitor with back and arm rests and were asked to watch the series of images presented at the screen during condition1 (constrained position) and condition 2 (body-head movement). The 39 face stimuli (13 faces $\times 3$ emotions) were presented in a randomized order and each stimulus was presented twice for $850 \mathrm{~ms}$ with a randomized duration of 500-1500 ms between two consecutive stimuli to avoid expectation effects. A fixation cross was randomly presented to the subjects to ensure that the subjects looked at the screen. EEG signals were recorded using the wireless Enobio system [14] with 19 channels according to the International 10-20 system with a sampling frequency of $500 \mathrm{~Hz}$.

\section{METHODS}

The EEG recordings were pre-processed with a band pass filter having a cut-off frequencies of $0.5 \mathrm{~Hz}-42 \mathrm{~Hz}$ and further processing involved the following stages - artifact reduction, epoching and feature extraction, explained in the following sections. An overview of the methodology has been illustrated in Figure 1. EEG data acquired from condition2 (contaminated by artifacts due to the subject's body-head movement and eye-blinking) was de-noised using an automated artifact reduction technique - WPT-EMD artifact reduction algorithm [9] prior to epoching. EEG epochs corresponding to each stimulus were then extracted from the pre-processed data for both conditions, obtaining an ensemble of 26 epochs for each emotional state (i.e. happy, fearful and neutral). A threshold of $200 \mu \mathrm{V}$ was applied on the 78 epochs $(26 \times 3$ emotions $)$ and the selected epochs within the thresholds were averaged to obtain the ERP data. These ERP data, from condition 1 and 2 were then used for the feature extraction.

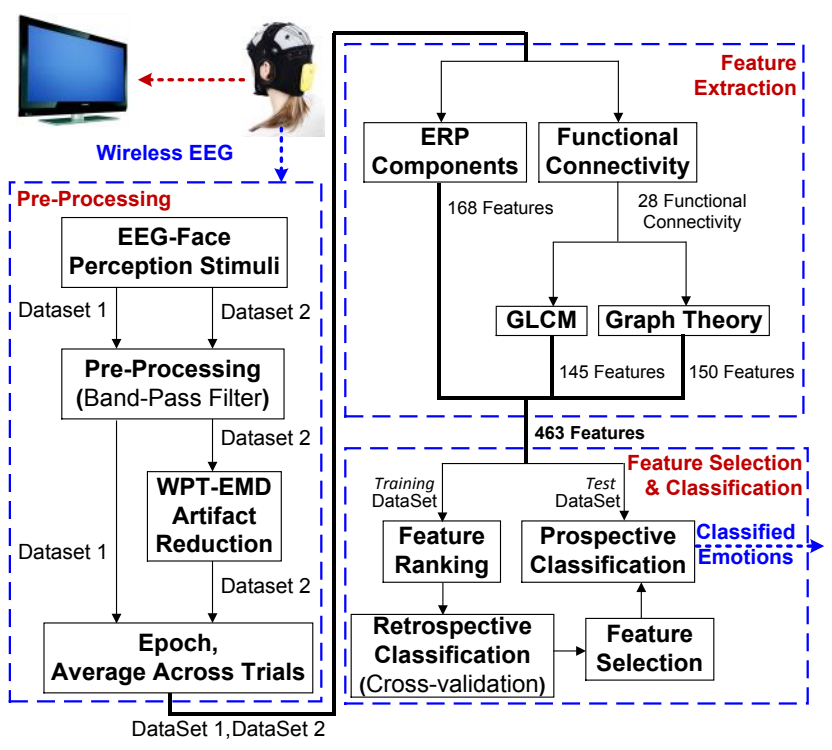

Figure 1. Overview of the methodology.

\section{A. Functional Connectivity (FC)}

Hermes Toolbox was used to generate the $29 F C$ measures; each of these is a matrix with size $19 \times 19$ (19 being the number of electrodes). Among the measures, described in Table I, four connectivity measures related to the phase synchronization $(P S)$ between two signals (i.e. 5-8) have been estimated for each individual band (in $\mathrm{Hz})-\theta(4-8), \alpha(8-12)$, $\beta$ (12-32), $\gamma(32-42)$ and all bands (6-42), resulting in total of $[(4 \times 5)+9] 29$ features.

TABLE I. Functional CONNECTIVITY MEASURES

\begin{tabular}{|l|l|l|}
\hline No. & \multicolumn{1}{|c|}{ Measures } & \multicolumn{1}{|c|}{ Description } \\
\hline 1. & CrossCorrelation & $\begin{array}{l}\text { linear correlation between two signals as a function } \\
\text { of time }\end{array}$ \\
\hline 2. & Correlation & Pearson's correlation coefficient (at zero lag) \\
\hline 3. & Coherence & $\begin{array}{l}\text { linear correlation between two signals as a function } \\
\text { of frequency }\end{array}$ \\
\hline 4. & $\begin{array}{l}\text { Phase Slope } \\
\text { Index }\end{array}$ & $\begin{array}{l}\text { estimation of the flow direction of information } \\
\text { between two signals as a function of time }\end{array}$ \\
\hline 5. & $\begin{array}{l}\text { Phase Locking } \\
\text { Value }(P L V)\end{array}$ & $\begin{array}{l}(P S) \text { inter-trial variability of the phase difference } \\
\text { between two signals at time } t\end{array}$ \\
\hline 6. & $\begin{array}{l}\text { Phase-Lag Index } \\
(P L I)\end{array}$ & $\begin{array}{l}(P S) \text { similar to PLV, however rejects phase } \\
\text { distributions centered around zero }\end{array}$ \\
\hline 7. & $\rho$ Index & $\begin{array}{l}(P S) \text { based on Shannon entropy, quantifies the } \\
\text { deviation of the distribution of the cyclic relative } \\
\text { phase from the uniform distribution }\end{array}$ \\
\hline 8. & $\begin{array}{l}\text { Directionality } \\
\text { Phase Indexes } \\
(D P I)\end{array}$ & $\begin{array}{l}\text { PS) Analysis of the temporal evolution of the } \\
\text { phase derivative }\end{array}$ \\
\hline 9. & $\begin{array}{l}\text { Granger } \\
\text { Causality }\end{array}$ & $\begin{array}{l}\text { linear parametric method, measures if signal } x \\
\text { provides predictive information about signal } y\end{array}$ \\
\hline 10. & $\begin{array}{l}\text { Transfer } \\
\text { Entropy }\end{array}$ & $\begin{array}{l}\text { is non-parametric, measures the amount of directed } \\
\text { information flow from signals } x \text { to } y\end{array}$ \\
\hline 11. & $\begin{array}{l}\text { Partial Directed } \\
\text { Coherence }\end{array}$ & $\begin{array}{l}\text { a frequency domain measure of Granger causality, } \\
\text { based on modelling time series by multivariate } \\
\text { autoregressive (MAR) processes }\end{array}$ \\
\hline 12. & $\begin{array}{l}\text { Direct Transfer } \\
\text { Function }\end{array}$ & $\begin{array}{l}\text { similar to PDC, however use a Hermitian transpose } \\
\text { instead of a Fourier transform }\end{array}$ \\
\hline 13. & $\begin{array}{l}\text { Mutual } \\
\text { Information } \\
\text { measures the amount of information shared }\end{array}$ \\
\hline
\end{tabular}

a. Detailed description of the measures have been provided in [7] 


\section{B. Feature Extraction: ERP Components}

ERP components related to emotion processing [6], described in Table II, are calculated for each electrode/region (central, frontal, occipital, parietal and temporal), resulting in $-a) 96$ features $[19$ electrodes +5 regions $\times(\mathrm{P} 100+\mathrm{N} 170) \times$ (amplitude, latency)]; b) 72 features [19 electrodes +5 regions $\times(\mathrm{P} 300+\mathrm{ESW}+\mathrm{LSW})]$, totaling 168 features.

TABLE II. ERP COMPONENTS

\begin{tabular}{|l|l|l|}
\hline No. & Measures & Description \\
\hline 1. & P100 & $\begin{array}{l}\text { reflects early sensory processing of visual } \\
\text { information; calculated for amplitude and latency }\end{array}$ \\
\hline 2. & N170 & $\begin{array}{l}\text { linked to sensitivity in processing information } \\
\text { from human faces; calculated for amplitude and } \\
\text { latency }\end{array}$ \\
\hline 3. & $\begin{array}{l}\text { linked to face recognition; calculated for mean } \\
\text { amplitude }\end{array}$ \\
\hline $4 / 5$ & $\begin{array}{l}\text { Early/Late Slow } \\
\text { Wave }(E S W / L S W)\end{array}$ & $\begin{array}{l}\text { linked to face processing and facial emotion } \\
\text { processing; calculated for mean amplitude }\end{array}$ \\
\hline
\end{tabular}

\section{Feature Extraction: Brain Connectivity Toolbox (BCT)}

$B C T$ applies graph theory analysis on the $F C$ measures except for 1, 3, 11, 12 (cf. Table I), since averaging across the thrid dimension of these $3 D$ matrices would negate the significance of these features, thereby yielding 150 features [25 FC×6].

TABLE III. GRAPH THEORETIC MEASURES

\begin{tabular}{|l|l|l|}
\hline No. & Measures & Description \\
\hline 1. & Transitivity & $\begin{array}{l}\text { measure of segregation (i.e. how many node's } \\
\text { neighbors are connected among themselves) }\end{array}$ \\
\hline 2. & Modularity & $\begin{array}{l}\text { measure of segregation; it measures how much the } \\
\text { network can be divided into subgroups with dense } \\
\text { links within-groups and few links between-group }\end{array}$ \\
\hline 3. & $\begin{array}{l}\text { Characteristic } \\
\text { path length }\end{array}$ & $\begin{array}{l}\text { measure of integration; measures the average } \\
\text { distance between nodes across the entire network }\end{array}$ \\
\hline 4. & $\begin{array}{l}\text { Global } \\
\text { efficiency }\end{array}$ & $\begin{array}{l}\text { measure of integration; it is the inverse of the } \\
\text { distance between nodes }\end{array}$ \\
\hline 5. & Radius & measure of shape of network-minimum eccentricity \\
\hline 6. & Diameter & measure of shape of network- maximum eccentricity \\
\hline
\end{tabular}

\section{Feature Extraction: GLCM}

GLCM measures the second order statistics to describe the distribution of the gray levels over the pixels in an image region. Here, it is used to quantify the $F C$ measures with a reduced dimensionality, resulting in 145 features [ $29 F C \times 5$ ].

TABLE IV. GLCM MEASURES

\begin{tabular}{|l|l|l|}
\hline No. & Measures & Description \\
\hline 1. & Contrast & measures the local variations of grey level \\
\hline 2. & Correlation & $\begin{array}{l}\text { measures the correlation between pixels in different } \\
\text { directions }\end{array}$ \\
\hline 3. & Homogeneity & measures the repetition of texture elements \\
\hline 4. & Entropy & measure of texture spatial disorder \\
\hline 5. & Energy & It is a measure of local homogeneity of the texture \\
\hline
\end{tabular}

This concludes the feature extraction process resulting in a total of 463 features extracted across ERP, BCT and GLCM. These features are correspondingly used for the next stage of classifying the emotional states.

\section{CLASSIFICATION}

We combine the feature sets from both the datasets (condition 1 and condition 2 ) thereby having a data matrix for 15 subjects, 463 features and three emotional states. The combined dataset is split into - a training dataset and a test dataset. The training dataset comprises of constrained EEG features of 7 subjects and movement EEG features of 5 subjects $(80 \%$ of data). The test dataset however comprises of only movement EEG features of 3 subjects ( $20 \%$ of data). Since in this work, our target was to classify the three emotional states from the movement EEG data, we opted to use only this in the test dataset. Using both constrained and movement EEG data in the training dataset helps to ensure a wider range of variability in the training set paving the way for a robust classification methodology which will produce acceptable levels of accuracy in a real-world application.

Prior to classification, we rank the features and select only the optimal number of features for dimensionality reduction. This is done using three steps -1 ) feature ranking using scatter matrices; 2) retrospectively classifying the training dataset using a 'leave-one-out' cross-validation strategy in conjunction with the ranked feature set using a SFS methodology to obtain the best combination of sequentially selected features; and 3) prospectively classifying the movement EEG test dataset.

We use the low-complexity class-separability measure based on scatter matrices to rank the 463 features. It ranks each individual feature for a multiple-class scenario where a high rank represents a small within-class variance and a large between-class distance among the data points in the respective feature space [15]. We follow the wrapper approach using the low-complexity SFS technique, selecting the first $i$ features of the ranked feature set in each iteration $(i$ $=1 \ldots 463$ ) of the retrospective classification in conjunction with a 'leave-one-out' cross-validation methodology on the training dataset. For this exploration, we restrict ourselves to two different classifiers - LDA and support vector machines (SVM), chosen from the perspective of using a low/moderate complexity classifier. SVM being a binary classifier in principle, we used the toolbox LIBSVM that is efficient for multi-class classification [16].

\section{RESULTS}

The retrospective classification using cross-validation in conjunction with feature selection helps to ascertain the best combination of features that resulted in highest accuracy for each individual classifier, calculated by averaging the individual accuracies across each cross-validation step for a sequentially selected feature combination. We achieve an overall accuracy of $81 \%$ using LDA (17 features) and 69\% using SVM (25 features) as a result of this stage. This selected feature set is further used for the next step of prospective classification on the movement EEG test dataset, which is the most important stage in any classification procedure, as it helps to determine the success of the crossvalidated model on the data that it has not been trained upon.

The results for prospective classification, using the best 
determined feature combination with the two classifiers on the test dataset comprising of three subjects and three emotional states are presented in Tables $\mathrm{V}$ and VI (illustrating the sensitivity for each class, overall accuracy considering a multi-class scenario and the best features). Using LDA and only 17 features (out of 463), we can successfully classify the three emotional states with sensitivities ranging between $(2 / 3$, i.e. $67 \%$ for neutral) to $(3 / 3$, i.e. $100 \%$ for emotional) and a total accuracy of $89 \%$.

TABLE V. SUMMARY OF SENSITIVITIES AND ACCURACY FOR THE MOVEMENT EEG DATA COLLECTED FROM 3 SUBJECTS

\begin{tabular}{|c|c|c|c|c|c|}
\hline \multirow[t]{2}{*}{ Classifier } & \multirow[t]{2}{*}{$\begin{array}{c}\text { No. of } \\
\text { Features }\end{array}$} & \multicolumn{3}{|c|}{$\begin{array}{l}\text { Sensitivity for each class } \\
\text { (true predictions out of } 3 \text { ) }\end{array}$} & \multirow[t]{2}{*}{$\begin{array}{c}\text { Accuracy } \\
(\%)\end{array}$} \\
\hline & & Happy & Fear & Neutral & \\
\hline LDA & 17 & 3 & 3 & 2 & 89 \\
\hline SVM & 25 & 3 & 3 & 1 & 78 \\
\hline
\end{tabular}

TABLE VI. LIST OF THE BEST FEATURES SELECTED IN THE CROSSVALIDATION STAGE FOR EACH CLASSIFIER

\begin{tabular}{|l|l|}
\hline Classifier & \multicolumn{1}{|c|}{ List of best Features } \\
\hline LDA & Transitivity[PLV( $\theta)]$; N170_Latency_F8; \\
& Transitivity[DPI $(\gamma)]$; LSW_MeanAmp_F7; \\
& LSW_MeanAmp_F4; ESW_MeanAmp_F7; \\
& ESW_MeanAmp_C4; Diameter[PLI $(\theta)]$; Energy[DPI $(\gamma)] ;$ \\
& N170_Amp_T8; N170_Amp_P3,CP_DPI, \\
& P300_MeanAmp_C4; P100_Amp_P3; \\
& ESW_MeanAmp_P8; N170_Amp [Cz,C4,C3]; \\
& N170_Latency_O2 \\
\hline SVM & Same as LDA features; Radius[PLI $(\theta)] ;$ \\
& LSW_MeanAmp_O1; P300_MeanAmp_P8; \\
& Correlation_DPI $\alpha$; P100_Latency_T7; N170_Amp_P8; \\
& Energy_PSI; P300_MeanAmp_O2 \\
\hline
\end{tabular}

An observation of the selected features for LDA shows the following significant features for each type of measure (cf. Table VI) - 1) all the ERP components in several channels or region (i.e. N170, LSW, ESW and P100); 2) among the functional connectivity measures, only the phase synchronization, specifically PLV and PLI in $\theta$ band and DPI in either $\gamma$ or all bands; 3 ) for the graph theoretic measures: transitivity (i.e. of $\operatorname{PLV}(\theta)$ and $\operatorname{DPI}(\gamma)$ ), diameter of $\operatorname{PLI}(\theta)$ and characteristic path length of DPI; 4) lastly, for GLCM measures only the energy (i.e. DPI $(\gamma)$ ) is the most significant feature. Hence, this helps us to determine the most significant features (17 out of 463) required to classify the target emotional states. Similarly, using SVM we achieve sensitivities in the range of $33-100 \%$, with an accuracy of $78 \%$ using 25 features having an inherent similarity with the top ranked features of LDA. However, there are few extra features required in this case, such as ERP components from additional channels (LSW O1), BCT (i.e. Radius[PLI $(\theta)]$ ) and $G L C M$ measures (i.e. Correlation[DPI $(\alpha)]$ ). The results using SVM are comparatively lower than LDA and the higher number of features required by the former, paves the way for LDA to be the most applicable classifier, besides being computationally less complex.

\section{DISCUSSION}

In this paper, we describe a systematic exploration using several EEG features to classify three emotional states pertaining to face perception. The processing methodology involved extracting a large number of neurophysiological features and effectively reducing the dimensionality using machine learning techniques. Our results show that we can successfully classify the three emotions using a simple LDA classifier using only 17 features chosen from ERP components and only three main parameters based on graph theory analysis extracted from phase synchronization- $F C$ measures. The three significant features from graph theory segregation (i.e. transitivity), integration (i.e. diameter) and shape of the network (i.e. $C P$ ) helps to further reduce the complexity of the feature extraction process, an essential step towards recognising the target emotions. The feature ranking/selection and classification techniques have been chosen considering the underlying computational complexity of the methodology. This paves the way for transforming the algorithms to a low-complexity implementation in software/hardware for supporting real-time emotion classification which can be applied for monitoring a wider subject population in the field of clinical neuroscience.

\section{REFERENCES}

[1] L. Carver and G. Dawson, "Development and neural bases of face recognition in autism.," Molecular Psychiatry, vol. 7, p. S18, 2002.

[2] A. Luneski, E. Konstantinidis, P. Bamidis, and others, "Affective medicine," Methods Inf Med, vol. 49, no. 3, pp. 207-218, 2010.

[3] A. Heraz and C. Frasson, "Predicting the three major dimensions of the learner's emotions from brainwaves," World Academy of Science, Engineering and Technology, vol. 31, pp. 323-329, 2007.

[4] I. B. Mauss and M. D. Robinson, "Measures of emotion: A review," Cognition and emotion, vol. 23, no. 2, pp. 209-237, 2009.

[5] Y.-Y. Lee and S. Hsieh, "Classifying different emotional states by means of EEG-based functional connectivity patterns," PloS one, vol. 9, no. 4, p. e95415, 2014.

[6] G. Chronaki, "A behavioural and electrophysiological exploration into facial and vocal emotion processing in children with behaviour problems," 2011.

[7] G. Niso, R. Bruña, E. Pereda, R. Gutiérrez, R. Bajo, F. Maestú, and F. del-Pozo, "HERMES: towards an integrated toolbox to characterize functional and effective brain connectivity," Neuroinformatics, vol. 11, no. 4, pp. 405-434, 2013.

[8] M. Rubinov and O. Sporns, "Complex network measures of brain connectivity: uses and interpretations," Neuroimage, vol. 52, no. 3, pp. 1059-1069, 2010.

[9] V. Bono, et al., "Artifact reduction in multichannel pervasive EEG using hybrid WPT-ICA and WPT-EMD signal decomposition techniques," in Acoustics, Speech and Signal Processing (ICASSP), 2014 IEEE International Conference on, 2014, pp. $5864-5868$.

[10] N. Aggarwal and R. Agrawal, "First and second order statistics features for classification of magnetic resonance brain images," 2012.

[11] M. Murugappan, R. Nagarajan, and S. Yaacob, "Comparison of different wavelet features from EEG signals for classifying human emotions," in Industrial Electronics |\& Applications, 2009. ISIEA 2009. IEEE Symposium on, 2009, vol. 2, pp. 836-841.

[12] S. S. Uzun, et al., "Emotion primitives estimation from EEG signals using Hilbert Huang Transform," in Biomedical and Health Informatics (BHI), 2012 IEEE-EMBS International Conference on, 2012, pp. 224-227.

[13] N. Tottenham, et al., "Behavioral assessment of emotion discrimination, emotion regulation, and cognitive control in childhood, adolescence, and adulthood," Frontiers in psychology, vol. 2, 2011.

[14] "Enobio." [Online]. Available: http://www.neuroelectrics.com/products/enobio/

[15] S. Theodoridis and K. Koutroumbas, "Pattern recognition and neural networks," in Machine Learning and Its Applications, Springer, 2001, pp. 169-195.

[16] "A Library for Support Vector Machines." [Online]. Available: http://www.csie.ntu.edu.tw/ cjlin/libsvm/. [Accessed: 2015] 